\title{
Clinical features of levodopa-induced dyskinesia in patients with parkinson's disease
}

\author{
Nargiza Mansurova, Anna Prokhorova. \\ Department of Neurology and Psychiatry at Bukhara state medical institute, \\ Department of Neurology at Tashkent medical academy.
}

\section{Email address:}

ayntraxt@gmail.com (Nargiza Mansurova, Anna Prokhorova.)

\section{To cite this article:}

Nargiza Mansurova, Anna Prokhorova. Clinical features of levodopa-induced dyskinesia in patients with parkinson's disease. Journal of research in health science. Vol. 2, No. 2, 2017, pp. 52-58. DOI 10.26739/2523-1243

\section{doij http://dx.doi.org/10.26739/2523-1243/-2017-2-2-9}

\begin{abstract}
In Parkinson's disease, one of the most troublesome dillemas is the treatment of levodopa-induced dyskinesia. The time-to-onset and severity of this motor complication show large variability according to disease duration, long-term use of levodopa and other factors. PD patients were enrolled to study based on UK brain bank diagnostic criteria and underwent examination on motor evaluation with help of UPDRS part III and presence of dyskinesia by UPDRS IV, to assess the severity of disease based on modified Hoehn and Yahr Scale. Considering the motor symptoms at onset, PD patients were divided into three subgroups: tremor- dominant, akinetic-rigid and mixed type. 208 patients $(105 \mathrm{men})$ were recruited in the study of who had advanced stage of PD. 105 $(50,4 \%)$ were classified as tremor-dominant, $87(41,9 \%)$ as akinetic- rigid and $16(7,7 \%)$ as mixed type. Patients in the tremor -dominant subtype were somewhat older $(\mathrm{p}=0,027)$. Approximately all patients were treated with L-dopa and $81,2 \%$ were in combination with dopamine agonist. Almost half patients received treatment with amantadine. In terms of disease duration and developing of wearing off, there was significantly difference according to clinical phenotype. AR and mix subtype of PD develop wearing off earlier within 3 years compare to TD $(\mathrm{P}<0,05) .72,4 \%$ TD type develop wearing off after 6 years and further while AR and Mix type develop earlier. (OR was 0.43; $95 \%$ CI 0.24-0.89, $R R$ was 0,69 ). It should be also noted that when gender was included in the analysis we didn't found a positive association with wearing off as well as LID (wearing off $=$ OR was 0,78 ; 95\% CI $0,43-1.41$; $\mathrm{p}$ value 0,$45 ; \mathrm{LID}=\mathrm{OR}$ was $0.73 ; 95 \% \mathrm{CI}$ 0.42-1.26; $\mathrm{RR}=0,85, \mathrm{p}$ value 0.27 ). Dyskinesias were predominantly peak dose in $64 \%$. The same no association with average LED with LID $(p=0,26)$. We may conclude that tremor dominant type of PD may predict slower progression of LID.
\end{abstract}

Keywords: motor fluctuation, levodopa-induced dyskinesia, advanced Parkinson's disease. 


\section{INTRODUCTION}

The cause of Parkinson's disease (PD) remains unknown, although a complex interaction among genetic and environmental factors is likely to be involved in the development and progression of the disease. The incidence rate was estimated to be 16,9 per 100 000 person-years in one Japanese study [1]. Levodopa remains the "gold-standard" anti parkinsonian drug to treat the motor symptoms of Parkinson's disease and, to this day, one of the successes of modern clinical pharmacology. However, its longterm use is associated with the development of levodopa-induced motor complications (MC), namely, motor fluctuations and dyskinesia.

Drug-induced dyskinesia is frequently the source of social and physical disability. These are indeed a considerable problem in the very young; among patients with PD onset before age 40 , both the dyskinesia-risk, and fluctuation-risk is over $95 \%$ after five years on levodopa $[2,3]$. However, more than $80 \%$ of PD patients present after age 60 , and in this age group, dyskinesia and fluctuation risks are markedly less. For those between ages 60-70, the dyskinesia risk after 5 years of levodopa is $26 \%$; it drops to $16 \%$ after age $70[4,7]$. Dyskinesia (DK) are defined as involuntary movements, the most common are chorea and dystonia, that can affect any body region, usually an extremity.[5]. This region can coincide with the first one affected by motor symptoms of PD. Initially they are peakdose related, but can also be dysphasic according to levodopa blood levels rise and fall - or "off dyskinesias" - when these levels are low[6]. Peak-dose dyskinaesias occur at the peak of benefit following the administration of levodopa, when patients are hypotonic and show only minimal signs of parkinsonism, and plasma levodopa levels are above a certain critical individual concentration [8]. They most commonly affect the upper part of the body, especially the face, the neck and the trunk, but they tend to be generalized, the upper limbs being more severely involved than the lower limbs [9].

Motor complications are important obstacles to the everyday life of PD patients. Therefore, the present study was designed to investigate a possible association between the specific phenotype of PD and development of LID, along with analysis of its targeted distribution and pattern.

Purpose: To study features of LID according to PD subtype and its somatotopic distribution in patients with Parkinson's disease.

Methods: This is descriptive and analytic study of a sample of 208 patients diagnosed with idiopathic PD who were consecutively recruited from the inpatient clinic of the movement disorders unit at Hospital University of Juntendo, Tokyo, Japan from 2006 to 2017 by using the medical records evaluated for deep brain stimulation (DBS). Diagnostic procedures at baseline included a full medical history, comprehensive clinical, neuropsychological and neuropsychiatric assessments, laboratory tests, cerebral MRI, and, if indicated clinically, dopamine transporter imaging and MIBG. Indication in patients with advanced PD include motor symptoms that have not responded to multiple treatments intended to control them, significant freezing episodes that limit daily activities, and non-motor complication, including 'off-time' anxiety attacks that negatively impact both the patient and family members. 


\section{Subject recruitments}

Eligible subjects were 29-81 years old and were diagnosed with advanced stage of Parkinson's disease as well as wearing off and dyskinesias of the limbs or trunk. Patients with multiple system atrophy, supranuclear palsy, vascular parkinsonism, drug-induced parkinsonism, and patients who had undergone deep brain stimulation surgery were excluded. Patients were subtyped into one of three clinical groups following the method proposed by Schiess and coworkers, The three subtypes were (1) akinetic-rigid (which includes postural instability, gait difficulty); (2) tremordominant; and (3) mixed (features of akinetic -rigid and tremor. Dyskinesias were recorded as choreic, atheoid, ballistic, dystonic or myoclonic character. Chorea was defined as random and chaotic flexion and extensions of the limbs. Chorea was often superimposed upon more repetitive, stereotypic dyskinesias. Dystonia was characterized by brief or sustained repetitive abnormal posturing associated with flexion or extension of the limbs.

\section{Standardized Instruments}

A motor examination was carried out, including the Unified Parkinson's Disease Rating Scale (UPDRS) part III examination. The total UPDRS part III score was used as a measure of disease severity (total score $=108$, higher score is worse). Motor complications were assessed using items of the UPDRS part IV. Only scores during the "on" medication state were included in the analysis. We used the modified Hoehn and Yahr Scale (five-point scale) to assess PD-related disabilities. Calculation of daily L-dopa equivalent daily dose (LEDD) was based on theoretical equivalence to L-dopa as follows: same anti-parkinsonian effect as 100 levodopa calculated for Ropinirole $x$ 9, Amantadine $\mathrm{x} 1$, pamipexole $\mathrm{x} 1$, Rotigotine $\mathrm{x} 4$, (all doses in $\mathrm{mg}$ ).

\section{Statistical analysis}

Statistical analysis was performed using GraphPad Prism 7 software for Mac. To gather all the information, a database was created using Microsoft Excel for Mac. Descriptive dataare presented as means, standard deviations (SDs) and percentages. The statistical significance among mean values in different groups and subgroups was determined by one and two way analysis of variance(ANOVA) followed by post hoc tests (Tukey's test). Between-group differences were compared using t-tests and ? 2 -tests as appropriate. Statistical threshold of *p $<$ $0.05, * * p<0.01$ and $* * * p<0.001$ were considered statistically significant.

Result: Finally 208 PD cases ( 105 men $(50,4 \%)$; mean age $65 \ddot{8} 8,51$ years) were included in the analysis. According to the symptom at onset of the 208 patients 105 $(50,4 \%)$ were classified as TD, 87 $(41,9 \%)$ as AR and $16(7,7 \%)$ as MT. Concerning the age at onset, out of the 208 PD patients $103(49,5 \%)$ had the onset of disease before the age 50 years. PD occurred before the age of 40 in $20 \%$ patients predominantly in male that have used the term "young onset". Patients in the tremor-dominant subtype were somewhat older $(\mathrm{p}=0.027)$. TD phenotype was significantly more common among the late onset, while AR was more frequent among the early onset $(\mathrm{p}<0,0001)$. There were no statistically significant differences in baseline HY stage, UPDRS, LEDD, disease duration in the comparison of PD subtypes. Baseline characteristics according to the clinical phenotype are shown in Table 1. 
Journal of research in health science. 2017; 2 (2): 52-58.

Table 1. Baseline characteristics of PD patients according to the clinical phenotype.

\begin{tabular}{|l|l|l|l|l|}
\hline & AR & TD & MT & \multirow{2}{*}{ v value } \\
\cline { 2 - 4 } & $\mathrm{N} \%$ & $\mathrm{~N} \%$ & $\mathrm{~N} \%$ & \\
\hline Gender & & & & \\
\hline men & $57(27,4 \%)$ & $40(19,2 \%)$ & $8(3,8 \%)$ & \\
\hline women & $48(23,1 \%)$ & $47(22,6 \%)$ & $8(3,8 \%)$ & NS \\
\hline Age & $63,6 \pm 9,59$ & $67,2 \pm 8,91$ & $65,3 \pm 7,03$ & $<0,027$ \\
\hline Duration & $15,9 \pm 5,22$ & $17,6 \pm 6,22$ & $15,3 \pm 5,30$ & NS \\
\hline Age at onset & $47,6 \pm 8,64$ & $49,5 \pm 9,36$ & $50,1 \pm 7,22$ & NS \\
\hline $\begin{array}{l}\text { Hoehn and Yahr } \\
\text { stage }\end{array}$ & $2,6 \pm 063$ & & & \\
\hline UPDRS III & $20.9 \pm 8,08$ & $21,9 \pm 8,43$ & $20,6 \pm 7,19$ & NS \\
\hline Average LED & $1065,9 \pm 371,82$ & $1007,6 \pm 304,67$ & $986,4 \pm 355,76$ & NS \\
\hline TR duration & $13,9 \pm 5,29$ & $15,3 \pm 6,63$ & $13,7 \pm 6,07$ & NS \\
\hline
\end{tabular}

Quantitative variable are expressed as mean \pm SD.AR= akinetic-rigid $P D, T D=$ tremor dominant PD, MT= mix type PD, UPDRS = Unified Parkinson's Disease Rating Scale; MMSE = MiniMental State Examination; LED $=$ Levodopa equivalent dose. NS=not significant $p>0.05$

In terms of disease duration and developing of wearing off, there was significantly difference according to clinical phenotype. AR and mix subtype of PD develop wearing off earlier within 3 years compare to TD $(\mathrm{p}<0,05)$. $72,4 \%$ TD type develop wearing off after 6 years and further while AR and Mix type develop earlier. (OR was 0.43; $95 \%$ CI 0.24-0.89, RR was 0,69). It should be also noted that when gender was included in the analysis we didn't found a positive association with wearing off as well as LID (wearing off= OR was 0,$78 ; 95 \%$ CI $0,43-1.41$; p value 0,45 ; LID $=$ OR was $0.73 ; 95 \%$ CI $0.42-$ $1.26 ; \mathrm{RR}=0,85, \mathrm{p}$ value 0.27 ). Dyskinesias were predominantly peak dose in $64 \%$. The same no association with average LED with LID $(p=0,26)$. (Table 2).75\% patients, dyskinesias started on the side of the most affected one of the onset of disease. Chorea was generally more severe in the legs than in the arms, characterized by chaotic alternating flexion and extensions of the limbs, with adduction and abduction of the shoulder and hip and circling and twisting movements of the hands and feet. The time of peak effect was between 60 and $140 \mathrm{~min}$ after treatment, coinciding with the time of peak locomotor activity. There is a developmental sequence of dyskinesia that usually began in the lower limbs and later involved the upper limbs and orofacial musculature.

Unlike chorea, dystonias were observed at the time of peak-dose dystonia and as drug-effects were diminishing end-of-dystonia. Young onset parkinsonian patients also develop dyskinesias restricted to the extremities, unlike older patients whose dyskinesias are more widespread, including the head and oral parts. 
Nargiza Mansurova, Anna Prokhorova. Clinical features of levodopa-induced dyskinesia in patients with parkinson's disease.

Table 2. LID and wearing-off prevalence according to PD subtypes and duration.

\begin{tabular}{|l|l|l|l|l|l|l|}
\hline & \multicolumn{2}{|l|}{ After 0-3 years } & \multicolumn{2}{l|}{ After 3-6 years } & \multicolumn{2}{l|}{ After 6 years } \\
\hline & WO & LID & WO & LID & WO & LID \\
\hline $\begin{array}{l}\text { TD } \\
(\mathrm{n}=87)\end{array}$ & $4(4,6 \%)$ & $3(3,4 \%)$ & $\begin{array}{l}27 \\
(31,4 \%)\end{array}$ & $\begin{array}{l}20 \\
(23,2 \%)\end{array}$ & $\begin{array}{l}55 \\
(63,2 \%)\end{array}$ & $\begin{array}{l}63 \\
(72,4 \%)\end{array}$ \\
\hline $\begin{array}{l}\text { AR } \\
(\mathrm{n}=105)\end{array}$ & $15(14,4 \%)$ & $7(6,7 \%)$ & $\begin{array}{l}44 \\
(42,3 \%)\end{array}$ & $\begin{array}{l}31 \\
(29,8 \%)\end{array}$ & $\begin{array}{l}45 \\
(43,2 \%)\end{array}$ & $\begin{array}{l}66 \\
(63,4 \%)\end{array}$ \\
\hline $\begin{array}{l}\text { Mix } \\
(\mathrm{n}-16)\end{array}$ & $4(26,6 \%)$ & $\begin{array}{l}4 \\
(26,6 \%)\end{array}$ & $6(37,5)$ & $2(12,5 \%)$ & $6(40 \%)$ & $\begin{array}{l}10 \\
(66,6 \%)\end{array}$ \\
\hline
\end{tabular}

\section{WO=wearing off, $\mathrm{LID}=$ levodopa induced dyskinesia}

Discussion: With continued levodopa therapy readily dyskinesia develops and it became more severe, more continues, and of longer duration. In patients with idiopathic Parkinson's disease of young onset, complications of therapy as a dyskinesia have been registered to emerge at an early stage in treatment. In three fourth patients, dyskinesias particularly affected those limbs which had initial parkinsonian symptoms, suggesting that the severity and location of the lesion are predisposing factors for dyskinesia. Peak dose dyskinesia most commonly affect the upper part of the body, especially the face, the neck and the trunk, but they tend to be generalized, the upper limbs being more severely involved than the lower limbs. They are predominantly choreic in nature but may also show dystonic features. Several studies have confirmed that patients with tremor at onset have a slower progression of disease than those with a AR subtype.[10,11,12]. Even if different risk factors have been suggested to be associated with the occurrence of dyskinesia in PD patients $[13,14]$, it is unclear whether patients with different clinical subtypes of PD differ in their risk of developing LID.

We evaluated the risk of od dyskinesia among TD and AR phenotypes and we have also found a decreased risk of developing dyskinesia among the TD subtype respect to the AR one, supporting the observation of a more benign course of PD in TD forms. In agreement with literature data and also in our sample, presence of LID was related to other wellrecognized risk factors such as disease duration, duration of dopaminergic drugs intake and average LED $[15,16]$.

Tremor-dominant patients show different morphologic lesion patterns compared to bradykinesia and rigidity dominant patients. The tremor-dominant type shows more severe cell loss in the medial substantia nigra (SN), which projects to the dorsolateral striatum and ventromedial thalamus, causing hyperactivity of thalamo motor and cerebellar projection. For the akineticrigid type, a more severe cell loss occurs in the ventro lateral part of $\mathrm{SN}$ and posterior putament, which causes inhibition of the glutamatergic thalamocortical pathway and reduced cortical activation. Different patterns of dopamine loss may reflect a variety of neuropathological features [17]. Tremordominant patients compared to akineticrigid patients showed a higher iodine-123 fluoropropyl-carbomethoxy (FP-CIT) uptake, which meant increased dopamine 
transportor (DAT) [18]. These data were confirmed by Eggers and colleagues during the clinical course and quantitative analyses of iodine-123 FP-CIT SPECT [19]. More recently, research on cerebrospinal fluid (CSF) revealed that PD patients who have postural instability and gait difficulties phenotype have reduced alfa-synucleinopathy amyloid-B markers levels compared to tremordominant patients [20]. Also, evidence suggested that low CSF amyloid-B might contribute to motor and cognitive decline in PD [21]. In this respect, tremordominant symptoms are related to a slower progress of PD.

The mechanism underlying the different types of dyskinesias remain largely unknown. Striatal cholinergic hyperfunction and a functional dopaminergic deficit, possibly involving only one subclass of dopamine receptors, have been proposed as the underlying mechanisms of foot dystonia in parkinsonism [22].

We noted that the age at the onset of PD was a strong determinant of LID: the occurrence of dyskinesia decreased with increased age of onset. One explanation is that younger PD patients have strong plasticity mechanisms, which leaves them at an extremely high risk of developing LID even with low doses of levodopa and mild dopamine depletion.[23]. As a previous study [24] suggested, chronic drug treatment and the severity of dopamine depletion associated with a long history of PD were major factors for LID. Our study demonstrated that the daily dose of levodopa, duration of disease and levodopa treatment ${ }^{\wedge}$ Hoehn-Yahr stage and UPDRS III score were related to the occurrence of LID. Our results support these findings. Overall, the significant risk factors for LID in multivariate logistic regression analysis were younger age at the onset of PD and the longer duration of levodopa therapy.

Dyskinesias usually appear first on the most affected side in asymmetrical parkinsonism, indicating that they are related to the severity of dopaminergic denervation. Dyskinesias are more frequent in patients with severe central dopaminergic depletion and with a good levodopa response [25,26,27].

Conclusion: Dyskinesias affected mostly those site which was affected at onset of disease. The risk of earlier developing of LID also depends on PD subtype along with other factors. TD type may predict not only slower progression, but also lower probability of developing LID. In the short run we hope to compare the data with the countries where not all group of antiparkinsonian drugs are available and to compare how does this effect in the occurrence of LID.

\section{References}

[1] Keiko Tanaka., Yoshihiro Miyake et al. (2011).Occupational risk factors for Parkinson's disease: a case-control study in Japan // BMC Neurology, 11:83

[2] Quinn N, Critchley P, Marsden CD. (1987).Young onset Parkinson's disease. Mov Disord 2:73-91.[PubMed]

[3] Schrag A, Ben-Shlomo Y, Brown R, Marsden CD, Quinn N.(1998). Young-onset Parkinson's disease revisited-clinical features, natural history, and mortality. Mov Disord 13:885-894. [PubMed] [4] Kumar N, Van Gerpen JA, Bower JH, Ahlskog JE. (2005).Levodopa-dyskinesia incidence by age of Parkinson's disease onset. Mov Disord 20:342-344. [PubMed] 
Nargiza Mansurova, Anna Prokhorova. Clinical features of levodopa-induced dyskinesia in patients with parkinson's disease.

[5] Thanvi BR, Lo TC. Long term motor complications of levodopa (2004). Clinical features, mechanisms, and management strategies. Postgrad Med J. 80:452-8

[6] Raudino F.(2012). The Parkinson disease before James Parkinson. Neurol Sci.33:945-8

[7] Ahlskog JE, Muenter MD.(2001). Frequency of levodopa-related dyskinesias and motor fluctuations as estimated from the cumulative literature. Mov Disord 16:448-58

[8] Muenter MD, et al. (1977). Patterns of dystonia ("i-D-I" and "D-I-D") in response to L-dopa therapy for Parkinson's disease. Mayo clinic Proc.

[9] Luquin MR, et al. (1992). Selective D2 receptor stimulation induces dyskinesia in parkinsonian monkeys, Ann Neurolo.

[10] Jankovic J, McDermott M, Carter J et al (1990) Variable expression of Parkinson's disease: a base-line analysis of the DATATOP cohort. The Parkinson Study Group. Neurology 40:1529-1534

[11] Jankovic J, Kapadia AS (2001) Functional decline in Parkinson disease. Arch Neurol 58:1611-1615

[12] Thenganatt MA, Jankovic J (2014) Parkinson disease subtypes. JAMA Neurol 71:499-504

[13] Manson A, Stirpe P, Schrag A (2012) Levodopa-induced-dyski- nesias clinical features, incidence, risk factors, management and impact on quality of life. J Parkinson Dis 21:189-198

[14] Olanow CW, Kieburtz K, Rascol O et al (2013) Factors predictive of the development of levodopa-induced dyskinesia and wearing- off in Parkinson's disease. Mov Disord 28:1064-1071

[15] Zappia M, Annesi G, Nicoletti G et al (2005) Sex differences in clinical and genetic determinants of levodopa peak-dose dyskinesias in Parkinson disease: an exploratory study. Arch Neurol 62:601-605

[16] Scott NW, Macleod AD, Counsell CE (2015) Motor complications in an incident Parkinson's disease cohort. Eur J Neurol.doi:10.1111/ene.12751

[17] Jellinger KA (2002) Recent developments in the pathology of Parkinson's disease. J Neural Transm Suppl 62:347-376

[18] C.Rossi, D.Frosini, D.Volterrani, P.De-Feo, E.Unti, V.Nicoletti, L.Kiferle, U.Bonuccelli, R.Ceravolo (2010). Differences in nigro-striatal impairment in clinical variants for early Parkinson's disease: evidence from a FP-CIT SPECT study, Eur.J. Neurol. 17 626-630.

[19] C.Eggers, D.J.Pedrosa，D.Kahraman, F.Maier, C.J.Lewis，G.R.Fink，M.Schmidt, L.Timmermann (2012). Parkinson subtypes progress differently in clinical course and imaging pattern, PLOS ONE7:1-8.

[20] G. Alves, K.F.Pedersen, B.R.Bloem, K.Blennow, H.Zetterberg, G.F.Borm, T.O.Dalaker, M.K.Beyer, D.Aarsland, U.Andreasson, J.Lange, O.B.Tysnes, R.Zivadinov, J.P. Larsen, (2013). Cerebrospinal fluid amyloid-B and phenotypic heterogeneity in de novo Parkinson's disease, J. Neurol. Neurosur. Psychiatry $84: 537-543$.

[21] M.Buongiorno, Y.Compta, M.J.Marti. (2011). Amyloid-? and ? biomarkers in Parkinson's diseasedementia, J. Neurol. Sci. 310:25-30.

[22] Poewe WH, et al., 1987.The pharmacology of foot dystonia in parkinsonism. Clin Neuropharmacolo, [23] G.Linazasoro (2005). New ideas on the origin of L-dopa-induced dyskinesias: age, genes and neural plasticity, Trends Pharmacol. Sci. $26: 391-397$.

[24] S.Fahn, D.Oakes, I.Shoulson, K.Kieburtz, A.Rudolph, A.Lang, C.W.Olanow, C.Tanner, K.Marek. (2004). Parkinson study group, levodopa and the progression of Parkinson's disease, N.Engl. J. Med. 351: 2498-2508

[25] Mones RJ, et al. 1971.Analysis of L-dopa induced dyskinesias in 51 patients with Parkinsonism. Neurol Neurosurg Psychiatry,

[26] Tomlinson CL, Stowe R, Patel S, Rick C, Gray R, Clarke CE (2010) Systematic review of levodopa dose equivalency reporting in Parkinson's disease. Mov Disord 25:2649-2653

[27] Kipfer S, Stephan MA, Schu pbach WM, Ballinari P, Kaelin-Lang A (2011) Resting tremor in Parkinson disease: a negative predictor of levodopa-induced dyskinesia. Arch Neurol 68:1037-1039 